\title{
Criação de bovinos em sistema silvipastoril com eucalipto: um risco para intoxicação por Ramaria flavo-brunnescens ${ }^{1}$
}

\author{
Daniel Machado Alves ${ }^{2}$, Clairton Marcolongo-Pereira ${ }^{3 *}$, Larissa Alt Tavares ${ }^{4}$, Kayane \\ R. Molarinho ${ }^{4}$, Margarida Buss Raffi ${ }^{3}$, Ana Lucia Schild ${ }^{3}$ e Eliza Simone V. Sallis ${ }^{3 * *}$
}

\begin{abstract}
Alves D.M., Marcolongo-Pereira C., Tavares L.A., Molarinho K.R., Raffi M.B., Schild A.L. \& Sallis E.S.V. 2014. [Cattle in silvipasture system with eucalyptus: a risk for Ramaria flavo-brunnescens poisoning.] Criação de bovinos em sistema silvipastoril com eucalipto: um risco para intoxicação por Ramaria flavo-brunnescens. Pesquisa Veterinária Brasileira 34(7):659-662. Laboratório Regional de Diagnóstico, Faculdade de Veterinária, Universidade Federal de Pelotas, Campus Universitário, Pelotas, RS 96010-900, Brazil. Email: esvsallis@yahoo.com.br

The aim of this study was to describe and discuss the epidemiological aspects of spontaneous outbreaks of poisoning by Ramaria flavo-brunnescens in cattle maintained in silvipasture system in Southern Brazil. Three outbreaks of the disease between 2011 and 2013 were diagnosed. In two outbreaks morbidity was 35\% and $37.4 \%$, and the mortality rate was $36.12 \%$ and $16 \%$, respectively. The fatality rate in these outbreaks was $45.71 \%$ and $96.55 \%$. The diagnosis was confirmed by the epidemiology and the presence of the mushroom in the areas where the cattle grazed, in addition to the clinical signs, gross and histological lesions. The climatic data of temperature, humidity and rainfall were statistically analyzed and no significant differences were observed between the years 2007-2013. It was not possible to confirm that the presence of $R$. flavo-brunnescens in eucalyptus groves is associated with rainy autumns and warm dry summers. It is likely that other factors are associated with the presence of the mushroom in the woods and its toxicity. The soil and type of forest planted may also determine the presence of the mushroom in the area. It is possible that the species of eucalyptus is also a determining factor for the occurrence of the mushroom, since in many eucalyptus forests $R$. flavo-brunnescens does not occur. The recognition of the mushroom by workers and farmers and the time for its development in the eucalyptus woods is essential to minimize economic losses caused by the poisoning.
\end{abstract}

INDEX TERMS: Poisonous plants, Ramaria flavo-brunnescens, Clavariaceae, silvo-pasture system, Eucalyptus spp., plant poisoning, mycotoxicosis, cattle.

RESUMO.- 0 objetivo deste trabalho foi descrever e discutir os aspectos epidemiológicos de surtos de intoxicação espontânea por Ramaria flavo-brunnescens em bovinos criados em sistema silvippastoril na região sul do Rio Grande do Sul. Foram diagnosticados três surtos da enfermidade

\footnotetext{
${ }^{1}$ Recebido em 6 de junho de 2014.

Aceito para publicação em 27 de junho de 2014.

${ }^{2}$ Programa de Pós-Graduação em Veterinária, Faculdade de Veterinária (FV), Universidade Federal de Pelotas (UFPel), Campus Universitário s/n, Pelotas, RS 96010-900, Brasil.

${ }^{3}$ Laboratório Regional de Diagnóstico, FV-UFPel. *Bolsista de Pós-Doutorado Júnior, CNPq (Proc. 150246/2014-5). ${ }^{* *}$ Autor para correspondência: esvsallis@yahoo.com.br

${ }^{4}$ Graduanda do Curso de Medicina Veterinária, FV-UFPel, Campus Universitário s/n, Pelotas, RS 96010-900.
}

entre 2011 e 2013. Em dois surtos a morbidade foi de $35 \%$ e $37,4 \%$ e a mortalidade foi de $36,12 \%$ e $16 \%$, respectivamente. A letalidade nos surtos foi de $45,71 \%$ e $96,55 \%$. 0 diagnóstico foi confirmado pela epidemiologia e presença do cogumelo nas áreas onde os bovinos estavam, além dos sinais clínicos e lesões macroscópicas e histológicas características. Os dados climáticos de temperatura, umidade e precipitação pluviométrica foram analisados estatisticamente; não se observou diferenças de 2007 a 2013. Não foi possível confirmar se a presença de R. flavo-brunnescens nos bosques de eucaliptos está associada a outonos chuvosos e quentes após verões secos. É provável que outros fatores estejam associados à presença do cogumelo nos bosques e à sua toxicidade. A influência do solo e a finalidade da mata plantada podem também, determinar a presença 
ou não do cogumelo na área. É provável que a espécie de eucalipto seja também um fator determinante para a ocorrência do cogumelo, já que em muitas matas deste gênero o vegetal $R$. flavo-brunnecens não ocorre. 0 reconhecimento do cogumelo por trabalhadores e produtores rurais e a época em que o mesmo se desenvolve nos bosques de eucalipto é fundamental para minimizar as perdas econômicas causadas pela intoxicação.

TERMOS DE INDEXAÇ̃̃O: Plantas tóxicas, Ramaria flavo-brunnescens, Clavariaceae, sistema silvipastorial, Eucalyptus spp., intoxicação por plantas, micotoxicose, bovinos.

\section{INTRODUÇÃO}

Ramaria flavo-brunnescens é um cogumelo da família Clavariaceae que se desenvolve somente em matas de eucalipto e tem sido descrito como tóxico para bovinos (Santos et al. 1975, Kommers \& Santos 1995, Barros et al. 2006), ovinos (Prucoli \& Camargo 1966), equinos (Santos et al. 1975, Barros et al. 2006) e suínos (Freitas et al. 1966). A intoxicação espontânea ocorre quando bovinos ingerem o cogumelo fresco que é altamente palatável (Santos et al. 1975). A morbidade pode chegar a $60 \%$ e letalidade varia de $20 \%$ a $50 \%$ (Bauer et al. 1966, Riet-Correa et al. 1985, Barros et al. 2006, Tokarnia et al. 2012).

As lesões macroscópicas e histológicas se restringem aos epitélios, especialmente aqueles que sofrem queratinização dura, como língua, pelos da vassoura da cauda e o estrato laminar do casco (Kommers \& Santos 1995, Schons et al. 2007).

O objetivo deste trabalho foi discutir os aspectos epidemiológicos de surtos de intoxicação espontânea por Ramaria flavo-brunnescens em bovinos criados em sistema silvipastoril na região sul do Rio Grande do Sul, alertando-se para os riscos do aumento da prevalência desta intoxicação em áreas reflorestadas com eucalipto.

\section{MATERIAL E MÉTODOS}

Estudou-se a epidemiologia de surtos de intoxicação por Ramaria flavo-brunnescens em bovinos ocorridos em uma área reflorestada com eucalipto no município de Jaguarão entre 2011 e 2013. Para a confirmação do diagnóstico da intoxicação três bovinos doentes foram eutanasiados in extremis e necropsiados, coletando-se fragmentos dos órgãos das cavidades abdominal e torácica, sistema nervoso central, casco, pele e língua, os quais foram fixados em formalina $10 \%$, processados rotineiramente e corados pela hematoxilina e eosina (HE).

A área reflorestada foi percorrida e foi investigado o tempo de implantação da mesma. Foram obtidos nos Boletins da Estação Agroclimatológica de Pelotas (Embrapa/UFPel 2014) os dados climáticos nos anos de 2007 a 2013 referentes à temperatura, umidade relativa do ar e precipitação pluviométrica nos meses de fevereiro, março abril, maio e junho. Estes dados foram analisados estatisticamente utilizando-se o teste não paramétrico de Kruskal-Wallis (GraphPad InStat 3.10).

\section{RESULTADOS}

Os surtos ocorreram em uma propriedade no município de Jaguarão, sul do Rio Grande do Sul, entre 2011 e 2013. No primeiro surto, de um lote de 155 bovinos, 58 ado- eceram $(37,4 \%)$ e 56 morreram $(36,12 \%)$; no segundo surto de 200 bovinos 70 (35\%) adoeceram e $32(16 \%)$ morreram. Os animais no primeiro surto eram machos, de 1,5 a 2,5 anos de idade e no segundo eram de categorias variadas. Os bovinos estavam numa área de 290 hectares reflorestada com Eucalyptus grandis e E. saligna (clone 2864), desde 2007 (Fig.1). Os bovinos, no primeiro surto foram colocados na área em novembro de 2009 e em abril de 2011 manifestaram sinais clínicos de intoxicação. No local havia grande quantidade de Ramaria flavo-brunnescens (Fig.1). No outono de 2012 casos da intoxicação foram relatados, porém o produtor desistiu do arrendamento da propriedade não sendo possível obter os dados epidemiológicos referentes ao surto. Em 2013 o surto ocorreu em maio e os bovinos estavam na área desde fevereiro. Em 2014 o proprietário recorreu a área e retirou os bovinos quando encontrou o cogumelo a partir do mês de abril. Os dados climáticos (temperatura, umidade relativa do ar e precipitação pluviométrica) não tiveram diferença significativa $(P>0,05)$ entre os anos analisados.

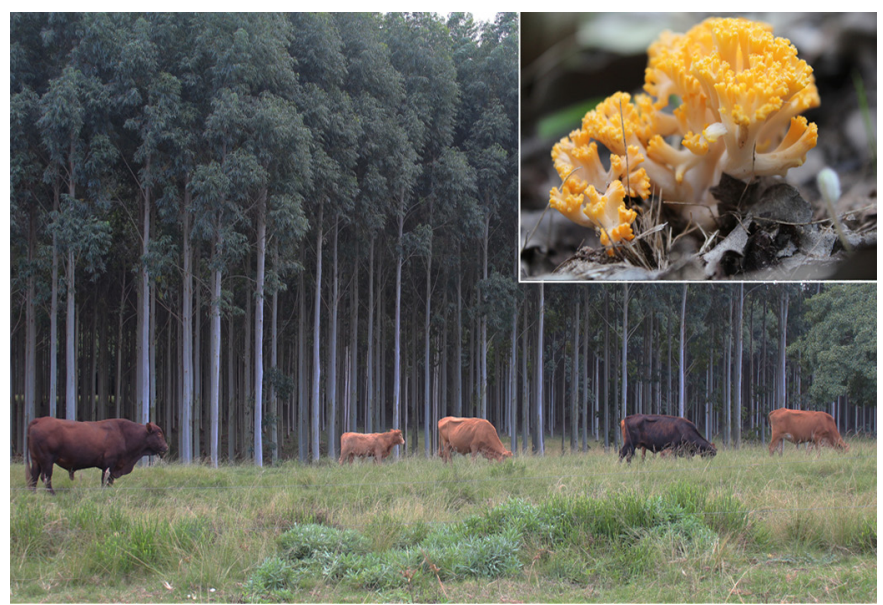

Fig.1. Bovinos pastoreando em sistema silvipastoril próximo ao bosque de Eucalyptus grandis onde a intoxicação por Ramaria flavo-brunnescens foi diagnosticada (Inset: Ramaria flavo-brunnescens desenvolvendo-se no solo do bosque de Eucalyptus onde o surto da intoxicação foi diagnosticado).

Os três bovinos necropsiados estavam em má condição corporal e os sinais clínicos observados caracterizavam-se por sialorreia, anorexia, secreção nasal e ocular e fezes escuras. Havia, ainda, afrouxamento e perda dos pelos longos da cauda, hemorragia da câmara anterior do globo ocular, opacidade da córnea e timpanismo. A evolução do quadro clínico foi de 4 a 10 dias.

Macroscopicamente havia ulceração dos bordos laterais e alisamento da superfície dorsal da língua, ulcerações no esôfago (Fig. 2) e alisamento das papilas ruminais; e ascite. Histologicamente havia atrofia do epitélio de revestimento e ulcerações na língua, as quais se estendiam até a lâmina própria. Nas áreas ulceradas havia infiltrado inflamatório de linfócitos, plasmócitos e macrófagos, além de tecido de granulação. No encéfalo havia, na região dorsal do óbex, área focal simétrica de malacia. 


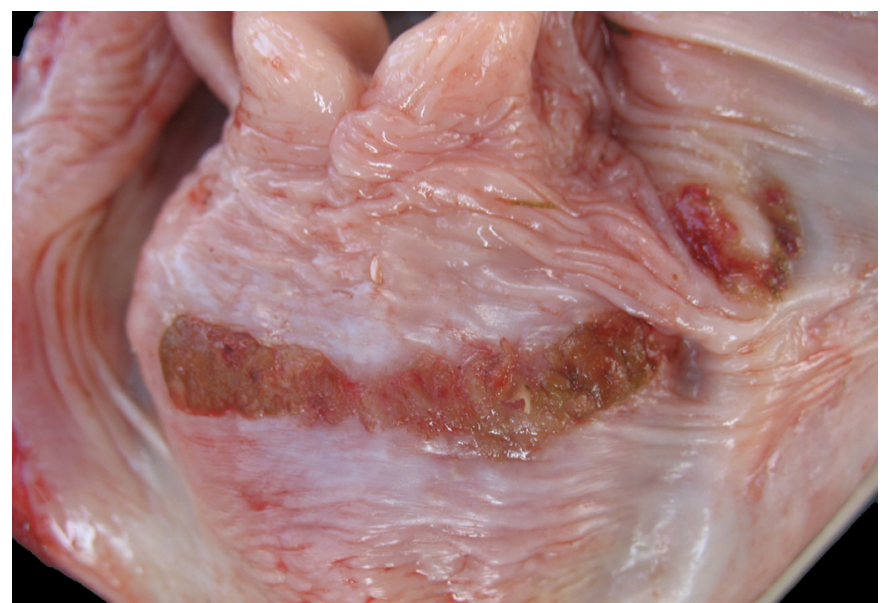

Fig.2. Lesão ulcerativa na porção inicial do esôfago de um bovino intoxicado por Ramaria flavo-brunnescens.

\section{DISCUSSÃO}

No presente estudo o diagnóstico de intoxicação por $R$. flavo-brunnescens foi realizado com base nos dados epidemiológicos, sinais clínicos, achados de necropsia e lesões histológicas. Os índices de morbidade e mortalidade foram similares aos índices mencionados em outros surtos da intoxicação descritos (Santos 1993, Barros et al. 2006, Tokarnia et al. 2012). A alta letalidade observada no surto de 2011 (96,55\%) poderia ser consequência de dois fatores principais: um, a grande quantidade do cogumelo presente onde os animais estavam, já que a espécie de eucalipto na área reflorestada era para produção de celulose e as árvores eram plantadas próximas umas as outras o que favorece o desenvolvimento do cogumelo (Fidalgo \& Fidalgo 1970, Crestana \& Moreira, 2009); e, outro o aumento da toxicidade do cogumelo. Variações na toxicidade foram evidenciadas em trabalhos experimentais realizados em ovinos em 2000 e 2004 utilizando o cogumelo de um mesmo bosque de eucaliptos (Sallis et al. 2004). No surto de 2013 o proprietário conhecia a intoxicação e os bovinos foram retirados da área após as primeiras mortes, o que provavelmente diminuiu a letalidade. Em 2014 não houve mortes porque os animais foram retirados assim que o cogumelo foi observado, além disso as áreas com maior quantidade foram cercadas.

No presente trabalho não foi possível confirmar que a presença de $R$. flavo-brunnescens nos bosques de eucaliptos está associada a outonos chuvosos e quentes após verões secos como é mencionado por alguns autores (Paschoal et al. 1983, Barros et al. 2006), já que não houve diferença significativa entre os dados climáticos analisados. É provável que outros fatores estejam associados à presença do cogumelo nos bosques e à sua toxicidade como fatores biogeoquímicos, dinâmica das comunidades vegetais e manutenção da estrutura do solo nestas áreas (Rillig \& Mummey 2006, Morris et al. 2009). A influência do solo e a finalidade da mata plantada podem, também, determinar a presença ou não do cogumelo na área. Por exemplo, matas nas quais os galhos secundários são cortados proporcionam um maior substrato de folhas no solo e poderiam levar ao desenvolvimento de maior quantidade de R. flavo-brunnescens. É provável que a espécie de eucalipto seja também um fator determinante para a ocorrência do cogumelo, já que em outros bosques deste gênero vegetal, na mesma região, $R$. flavo-brunnecens não tem sido observada.

A intoxicação por $R$. flavo-brunnescens em áreas de reflorestamento com o eucalipto chama a atenção para o sistema silvipastoril de criação de animais de produção, o qual se caracteriza pela combinação intencional de árvores, pastagem e ruminantes numa mesma área ao mesmo tempo com um manejo integrado, objetivando o aumento da produtividade e a sustentabilidade (Porfírio-da-Silva, 2004). A possível ocorrência da intoxicação por este cogumelo já havia sido mencionada em boletins e comunicados técnicos da Emater e Embrapa neste sistema de criação (Embrapa 2005, Ferro 2012).

0 diagnóstico de surtos em anos subsequentes em uma área de reflorestamento com a morte de pelo menos 88 bovinos $(24,79 \%)$ de 355 animais sob risco demonstra que a intoxicação pode trazer prejuízos econômicos importantes para a região Sul do Rio Grande do Sul. Esta região foi a que apresentou o maior aumento percentual (42\%) de florestas de eucalipto plantadas em áreas próprias (ABRAF 2010) e atualmente a utilização dessas áreas para manutenção de bovinos e ovinos é uma realidade. 0 reconhecimento do cogumelo por trabalhadores e produtores rurais e a época em que o mesmo se desenvolve nos bosques de eucalipto é fundamental para minimizar as perdas econômicas causadas pela intoxicação.

\section{REFERÊNCIAS}

ABRAF 2010. Florestas plantadas no Brasil. Florestas plantadas com eucalipto e pinus. Disponível em <www.abraflor.org.br/estatísticas/ ABRAF10-BR/capítulo01.pdf> Acesso em 14 mai. 2014.

Barros R.R., Irigoyen L.F., Kommers G.D., Rech R.R., Fighera R.A., Barros C.S.L. 2006. Intoxicação por Ramaria flavo-brunnescens (Clavariaceae) em bovinos. Pesq. Vet. Bras. 26:87-96.

Bauer A.G., Laranja R.J. \& Santos A.G. 1966. Sobre a etiologia do chamado "mal do eucalipto". Arq. Inst. Pesq. Vet. Desidério Finamor 3:85-90.

Crestana M.S.M. \& Moreira R. 2009. Plantio de eucalipto. Disponível em <http://www.cati.sp.gov.br/Cati/_tecnologias/culturas_florestais/ plantio_eucalipto.php> Acesso em 14 mai. 2014.

Embrapa 2005. Sistemas silvipastoris: árvores e pastagens, uma combinação possível. Embrapa Gado de Corte, Campo Grande, MS. Disponível em <http://saf.cnpgc.embrapa.br/publicacoes/arvoresepastagens.pdf> Acesso em 14 mai. 2014.

Embrapa/UFPel 2014. Boletim Agroclimatológico. Estação Agroclimatológica de Pelotas. Disponível em <http://www.cpact.embrapa.br/agromet/ estacao/ boletim.html> Acesso em 14 mai. 2014.

Ferro J.X. 2012. Intoxicação de bovinos e ovinos por cogumelo. Emater. Disponível em <http://www.emater.go.gov.br/w/4644> Acesso em 14 mai. 2014.

Freitas J., Pasturino C.L., Quiñones-Sowerby C.A., Bellagamba C., Giambruno E., Infantozzi J.M., Decia J.C. \& Gerveñanzky W. 1966. “Comunicacion sobre uma enfermedad aparecida em ganados del Uruguay em los últimos años (BOCOPA)" 5 Congresso Panamericano de Medicina. Veterinaria y Zootecnia, Caracas, p.152-159.

Fidalgo O. \& Fidalgo M.E.P.K. 1970. A poisonous Ramaria from southern Brazil. Rickia, USA, 5:71-91.

GraphPad InStat. Graphpad software. Version 3.10. San Diego, 2013. Diponível em <http://www.graphpad.com/scientific-software/instat/> Acesso em 14 mai. 2014. 
Kommers J.D. \& Santos M.N. 1995. Experimental poisoning of cattle by the mushroom Ramaria flavo-brunnescens (Clavariaceae): a study of the morphology and pathogenesis of lesions in the hooves, tail, horns, and tongue. Vet. Human Toxicol. 37:297-302.

Morris M.H., Pérez-Pérez M.A., Smith M.E. \& Bledsoe C.S. 2009. Influence of host species on ectomycorrhizal communities associated with two cooccuring oaks (Quercus spp.) in a tropical cloud forest. FEMS Microbiol. Ecol. 69(2):274-287.

Paschoal J.P., Portugal M.A.S.C. \& Nazário W. 1983. Ocorrência do "mal do eucalipto em bovinos no estado de São Paulo. Biológico 49(1):15-18.

Porfírio-da-Silva V. 2004. Sistemas silvipastoris. Centro Nacional de Pesquisa de Florestas. Disponível em <http://www.cnpf.embrapa.br/pesquisa/safs/> Acesso em 14 mai. 2014.

Prucoli J.O. \& Camargo W.V.A. 1966. Intoxicação experimental em ovinos com Clavaria spp. Boletim Industrial Animal, Nova Odessa, 23:177-178.

Riet-Correa F., Schild A.L., Méndez M.C., Brod C.S. \& Ferreira J.L.M. 1985. Intoxicação por Ramaria flavo-brunnescens em bovinos. Laboratório Regional de Diagnóstico. Doenças diagnosticadas no ano 1984. Editora Universitária, Pelotas, p.28-29.
Rillig M.C. \& Mummey D.L. 2006. Tansley review: mycorrhizas and soil structure. New Phytol. 171:41-53.

Sallis E.S.V., Raffi M.B. \& Riet-Correa F. 2004. Intoxicação experimental em ovinos com Ramaria flavo-brunnescens congelada ou dessecada. Pesq. Vet. Bras. 24:107-110.

Santos M.N., Barros S.S. \& Barros C.S.L. 1975. Intoxicação em bovinos pelo cogumelo Ramaria flavo-brunnescens. Pesq. Agropec. Bras., Ser. Vet. 10:105-109.

Santos M.N. 1993. Intoxicação por Ramaria flavo-brunnescens, p.309-318. In: Riet-Correa F., Méndez M.C. \& Schild A.L. (Eds), Intoxicações por Plantas e Micotoxicoses em Animais Domésticos. Editorial Agropecuária Hemisfério Sul do Brasil, Pelotas. 340p.

Schons S.V., Kommers G.D., Pereira G.M., Raffi M.B., Schild A.L. 2007. Estudo histológico, imunohistoquímico e ultra-estrutural das lesões induzidas experimentalmente por Ramaria flavobrunnescens (Clavariaceae) em bovinos. Pesq. Vet. Bras. 27(7):269-276.

Tokarnia C.H., Brito M.F., Barbosa J.D., Peixoto P.V. \& Döbereiner J. 2012. Plantas Tóxicas do Brasil para Animais de Produção. 2aㅡ ed. Editora Helianthus, Rio de Janeiro. 566p. 